\title{
Corresponsabilidad familiar: negociación e intercambio en la división del trabajo doméstico
}

\author{
M. ${ }^{a}$ del Carmen Rodríguez Menéndez \\ José Vicente Peña Calvo \\ Susana Torío López \\ Universidad de Oviedo. Departamento de Ciencias de la Educación \\ carmenrm@uniovi.es \\ vipe@uniovi.es \\ storio@uniovi.es
}

Recibido: 26-02-2008

Aceptado: 23-06-2009

\section{Resumen}

En el presente artículo, mostramos los resultados de una investigación cualitativa realizada en el Principado de Asturias para conocer el reparto de tareas domésticas en las familias asturianas. A través del uso de grupos de discusión, analizamos las actitudes, las preferencias y las creencias que poseen hombres y mujeres acerca de la corresponsabilidad familiar. Además, también analizamos en qué medida están marcadas por el estereotipo de género. Si bien aquí no presentamos, de forma exhaustiva, todas las conclusiones obtenidas, exponemos los resultados referidos a los procesos de negociación que dominan el reparto de funciones y responsabilidades familiares.

Palabras clave: división sexual del trabajo, género, grupos de discusión.

\section{Abstract. Familiar co-responsibility: negotiation and exchange in the division of domestic} labor

This paper presents the results of a qualitative research study in the Principality of Asturias on current sharing of domestic tasks in Asturian families. Using focus groups, we show men and women's attitudes, preferences, and beliefs about family shared responsibilities. We also analyze to what extent those attitudes are influenced by gender stereotypes. Although we do not present exhaustively all the findings of the research, we discuss specific conclusions about negotiation and exchange processes that dominate the sharing of domestic labor.

Key words: sexual division of labor, gender, focus group.

\section{Sumario}

1. La división sexual del trabajo doméstico

2. Diseño de la investigación
3. Resultados obtenidos: los procesos de acuerdo y negociación en el reparto de las tareas domésticas

4. Discusión de los resultados

5. Referencias bibliográficas 


\section{La división sexual del trabajo doméstico}

La abundante investigación cuantitativa que se ha realizado a lo largo de las décadas pasadas ha contribuido a conocer, de forma exhaustiva, el fenómeno de la división sexual del trabajo doméstico. La atención por el estudio de este fenómeno ya se inició en el ámbito anglosajón, en particular en los EEUU, durante la década de 1960 y culminó en la de 1990, cuando creció, de forma exponencial, el número de libros y artículos publicados sobre dicho tema. En nuestro país, los primeros estudios que tienen rigor científico datan de la década de 1980. Entre ellos, destaca el trabajo pionero titulado De puertas adentro, de M. A. Durán.

En muchos de los estudios emprendidos, el concepto de trabajo doméstico no se ha definido de forma explícita. En nuestro caso, consideramos necesario conceptuar lo que entendemos por dicha expresión. Para ello, partimos de la definición proporcionada por Shelton y John, para quienes el trabajo doméstico es «aquel trabajo no remunerado que contribuye al bienestar de los miembros del grupo familiar y al mantenimiento del hogar» (Shelton y John, 1996: 300. La traducción es nuestra).

En este contexto, dividimos el trabajo doméstico en tres categorías fundamentales:

1. Tareas del hogar: que incluye aquellas labores relativas a la administración de recursos y al consumo familiar, la limpieza de la casa, la preparación de alimentos, el transporte, la representación y las relaciones en el exterior, así como las tareas de reparación y mantenimiento de la vivienda.

2. Cuidado de los hijos e hijas y de otras personas dependientes: que incluye todas las actividades implicadas en la atención a ellas, tales como, por ejemplo, darles de comer, vestirlas, llevarlas al médico, darles las medicinas, proporcionarles transporte y acompañamiento, etc.

3. Trabajo emocional: nos referimos a aquellas actividades que permiten mejorar el bienestar y proveer soporte emocional a otras personas. Ello supone que dicho concepto incluye actividades como dar apoyo, escuchar atentamente, saber hacer ver a los miembros de la familia que se los aprecia y se siente cariño por ellos, etc.

En este contexto, son muchas las investigaciones que constatan que la mayoría de la población mantiene una opinión favorable a que las tareas domésticas se realicen de forma equitativa por ambos miembros de la pareja. Las preferencias de los españoles y de las españolas por un modelo de familia igualitaria han aumentando considerablemente en los últimos tiempos, de modo que, si bien a finales de la década de 1980 y principios de la de 1990, cerca del $47 \%$ de los españoles se identificaban con ese ideal de familia, en la actualidad lo hace más del 60\% (Navarro, 2006. Véase también Alberdi, 1999; Alberdi y Escario, 2007; Valiente, 1997).

Sin embargo, los estudios también son unánimes al declarar que si bien se ha producido un cambio de actitudes, éste no ha venido acompañado de una 
modificación sustancial de las conductas que hombres y mujeres desempeñan en sus hogares (Alberdi, 1999; Brullet, 1996; Papí y Frau, 2005; Pérez-Díaz, Chulia y Valiente, 2000; Subirats, 1993; Torns, Borrás y Carrasquer, 2003-2004; Valiente, 1997). Aunque se indica que la división del trabajo doméstico debe estar distribuida a partes iguales, la realidad dista de asemejarse a este modelo ideal percibido. Por tanto, si bien las mujeres hacen menos trabajo doméstico y los hombres hacen un poco más ahora que hace veinte años, siguen siendo ellas las que invierten mucho más tiempo en este tipo de tareas. Por tanto, la corresponsabilidad familiar, entendida como una distribución de las labores y las responsabilidades domésticas que sea equitativa y que implique por igual a todos los miembros del grupo familiar, todavía no se ha logrado.

Asimismo, el análisis de los artículos científicos permite comprender las causas que explican la distribución desigual del reparto de las tareas domésticas (véase Almeida, Maggs y Galambos, 1993; Apparala, Reifman y Munsch, 2003; Bartau, Maganto y Etxeberría, 2002; Baxter, Hewitt y Western, 2005; Bianchi, Milkie, Sayer y Robinson, 2000; Cunningham, 2005; Davis y Greenstein, 2004; Greenstein, 1996a, 1996b; Kroska, 2004; Meil, 1997a, 1997b; Mikula, 1998; Pittman y Blanchard, 1996; Presser, 1994; Sánchez y Thomson, 1997; South y Spitze, 1994; Stevens, Kiger y Riley, 2001; Sullivan, 2000, 2004; Twiggs, McQuillan y Marx, 1999). En particular, se han desarrollado tres teorías fundamentales que permiten explicar y comprender las causas que originan la ausencia de un reparto equilibrado:

1. La teoría de los recursos, que estima que el cónyuge que dispone de más recursos tiene más poder y, por tanto, hará menos trabajo reproductivo.

2. Las explicaciones basadas en la ideología de género, que constatan que hombres y mujeres con una ideología de género tradicional se acomodarán a un reparto más desequilibrado de las tareas domésticas. Asimismo, aquellas mujeres y hombres que manifiesten actitudes de género más abiertas, tenderán a repartirse este trabajo de forma más equitativa.

3. La teoría de la disponibilidad temporal: aquellas personas que invierten más tiempo en el trabajo productivo, dedican menos tiempo a la realización de las tareas domésticas.

A su vez, también se han analizado otros factores que influyen en la división del trabajo doméstico, tales como la edad de los cónyuges (Apparala, Reifman y Munsch, 2003; Bartau, Maganto y Etxeberría, 2002; Bianchi, Milkie, Sayer y Robinson, 2000), el estatus marital (Baxter, Hewitt y Western, 2005; Bianchi, Milkie, Sayer y Robinson, 2000; Coltrane, 1996, 2000; Cunningham, 2005; Kroska, 2004; South y Spitze, 1994) o la edad y la presencia de niños y niñas en el hogar (Bartau, Maganto y Etxeberría, 2002; Bianchi, Milkie, Sayer y Robinson, 2000; Coltrane, 2000; Cunningham, 2005; Davis y Greenstein, 2004; Kroska, 2004; Presser, 1994; Sánchez y Thomson, 1997).

En consecuencia, el desarrollo teórico obtenido es de gran interés, porque explica cuales son los factores que influyen en la división del trabajo domés- 
tico y el papel relativo que desempeña cada uno de ellos en la explicación causal del fenómeno. Sin embargo, en el caso del presente artículo estudiamos el proceso que siguen las parejas para negociar la distribución de tareas, asunto que no ha sido analizado con detalle por las investigaciones realizadas.

Entendiendo el término negociación como el proceso por el que dos o más personas buscan llegar a un acuerdo sobre un asunto determinado, hemos de considerar que las parejas intentan negociar los términos del reparto doméstico para lograr una mejor conciliación de la vida laboral y familiar. En este contexto, asumimos que la división del trabajo doméstico no es un acuerdo fijo y estático entre los integrantes del grupo familiar, sino que se debe analizar como un proceso en constante negociación y, por consiguiente, sujeto a profundas transformaciones a lo largo del ciclo vital familiar (Deutsch, 1999; Doucet, 1995, 2001; Kluwer, 1998; Zvonkovic, Greaves, Schmiege y Hall, 1996).

Las parejas configuran el reparto a partir de la acumulación de muchas y pequeñas decisiones y actos que acontecen en su vida familiar. Hombres y mujeres no asumen de forma pasiva sus respectivas responsabilidades domésticas, sino que lo que se decide hacer o no hacer se negocia y se renegocia a través de la interacción marital, la cual va definiendo las categorías de lo posible y deseable para cada uno de los géneros. Esto significa que la división del trabajo doméstico nunca queda totalmente cerrada, sino que está continuamente recreada a partir de los cambios que acontecen en la vida cotidiana.

\section{Diseño de la investigación}

\subsection{Objetivo de la investigación y enfoque metodológico}

Hemos adoptado un enfoque de investigación cualitativo que permite comprender los procesos por los que las parejas reparten las tareas domésticas, analizando las decisiones establecidas y cómo esas decisiones permiten mantener o rechazar los estereotipos de género. Diversos autores y autoras (Deutsch, 1999; Doucet, 2001; Matthews, 2005; Sánchez, 1994; Zvonkovic, Greaves, Schmiege y Hall, 1996) indican la necesidad de realizar estudios cualitativos, basados en el uso de entrevistas en profundidad y grupos de discusión, que animen a hombres y mujeres a reflexionar y discutir sobre los procesos de corresponsabilidad familiar. Las personas tienen historias que contar acerca de sus experiencias en los procesos de negociación familiar, y esas historias gozan de una gran potencia explicativa, por lo que se precisan aproximaciones cualitativas que las revelen.

En el campo de estudio referido a la corresponsabilidad familiar han abundado las investigaciones cuantitativas. Sin desestimar este tipo de investigaciones, es necesario realizar estudios cualitativos que analicen los discursos de las personas implicadas en los procesos de distribución de las responsabilidades domésticas. 


\subsection{Instrumentos de recogida de datos y selección de contextos y casos}

Se tomó la decisión de usar la técnica del grupo de discusión por varias razones. En primer lugar, esta técnica provee el contexto adecuado para que hombres y mujeres dialoguen sobre los procesos de negociación y discusión generados por el reparto doméstico. Los discursos provocados en el seno del grupo nos permiten comprender la distribución del trabajo familiar en función del género, entendiendo que éste es un fenómeno socialmente construido y estructuralmente constreñido. Además, el grupo de discusión permite crear un ambiente en el que los distintos participantes son estimulados a intervenir activamente en el diálogo por las ideas y las opiniones que presentan los demás, incrementando la calidad de los datos recogidos. Por último, es una técnica muy efectiva para obtener información acerca de las percepciones, los sentimientos, las creencias y las actitudes de las personas participantes en la investigación (Krueger, 1991).

Se organizaron nueve grupos de discusión y participaron en ellos un total de cincuenta y seis personas. Se realizaron tres sesiones en el centro de Asturias, que se completaron con dos visitas a las zonas de las cuencas mineras. A su vez, se efectuaron cuatro reuniones en las zonas del oriente (dos) y del occidente (dos) asturiano. Cada uno de los grupos tuvo una duración aproximada de dos horas y la media de participantes fue de seis personas.

Definimos nuestra población objeto de estudio como hombres y mujeres asturianos que han formado familias en las que hay, al menos, un hijo o una hija en edad escolar. Por ello, todas las parejas participantes tenían descendencia. Para determinar la composición de los grupos, atendimos a los siguientes criterios:

1. Lugar de residencia: formamos grupos de discusión con familias que vivían en diversas zonas geográficas del Principado de Asturias, acudiendo a ámbitos urbanos y rurales.

2. Tipología y estructura familiares: para atender al criterio de diversidad, establecimos la conveniencia de que participasen diversas tipologías familiares. Por ello, había parejas casadas en segundas nupcias, familias monoparentales, parejas de hecho con hijos e hijas, parejas casadas con hijos e hijas, etc. No se incluyeron parejas homosexuales porque no hubo la oportunidad de contactar con ellas.

3. Presencia de los dos miembros de la pareja en la sesión de grupo: de esta forma, el contexto de la discusión se veía enriquecido porque las afirmaciones realizadas por uno de los miembros de la pareja eran complementadas o rebatidas con las aportaciones que hacía el cónyuge.

Del total de participantes, el $57,1 \%$ está formado por mujeres y el $42,9 \%$, por varones, por lo que hay una ligera sobrerrepresentación femenina, que es debida a que las familias monoparentales han estado representadas por mujeres. En cuanto al nivel de estudios, el 33,9\% de las personas declara que tiene 
estudios primarios; seguido por un $21,4 \%$ de licenciados universitarios, que sumado al $12,5 \%$ de diplomados universitarios da una total coincidencia porcentual con respecto a los que tienen estudios primarios, a saber: un 33,9\%. Les siguen aquellos que disponen de estudios de bachillerato o de formación profesional, de modo que, sumando ambas casillas, obtenemos un $28,5 \%$.

\subsection{Procedimiento}

Nuestra formación profesional como pedagogos nos ha inclinado a elegir las familias colaboradoras a partir de nuestros contactos con profesionales que trabajan en el contexto escolar. Nos propusimos explotar nuestras redes sociales (docentes, equipos directivos, AMPA, etc.) para plantearles nuestra investigación e ir afianzando las relaciones de confianza necesarias para abordar el objeto de estudio. En este sentido, recurrir a instituciones educativas para contactar con familias es una práctica habitual en la investigación socioeducativa. Tras varios intentos de selección inicial de las familias, fueron las asociaciones de madres y padres las que mostraron más interés por la investigación y quienes fueron las encargadas de organizar los grupos atendiendo a los requerimientos muestrales establecidos.

Durante las sesiones de grupo usamos un guión de preguntas, reelaborado después de la primera sesión, que facilitó la organización de la información y el análisis de contenido realizado con posterioridad. Dado que lo que nos interesaba era explorar los esquematismos de género, el guión de preguntas fue una guía abierta que no condicionó el desarrollo de las sesiones. No nos importaba que todos los tópicos fueran trabajados en todos los grupos, sino lograr un clima que favoreciese el debate para desvelar las preconcepciones que guiaban el ejercicio de la negociación sobre el reparto de las tareas domésticas.

Algunas de las preguntas que orientaron la discusión fueron las siguientes: «¿Cómo es vuestro reparto de las tareas domésticas?», «¿Cómo negociáis el reparto?», "¿Ha cambiado el reparto de tareas a lo largo de vuestra vida en común?», "¿Alguna vez habéis discutido sobre el reparto de tareas?», "¿Podéis ponernos algún ejemplo?», «¿Cómo solucionáis los conflictos?», etc.

Aunque los participantes conocían el fin para el que habían sido convocados, las reuniones comenzaron con una exposición de nuestros objetivos. Presentábamos nuestra investigación como un estudio diagnóstico sobre los procesos de división del trabajo doméstico en el Principado de Asturias. Les comentábamos nuestro interés en analizar el reparto de tareas que existía en su hogar, así como los procesos de negociación que habían provocado el reparto que tenían. Este fue el hilo argumentativo usado, de modo que la idea fue plantear la investigación de la manera más amplia posible, evitando la preparación de discursos alejados de la realidad. Además, intentamos transmitir una valoración positiva de sus aportaciones y dejamos claro que nuestro interés no era juzgar su reparto de tareas. Aclaramos que lo que se dijera, además de ser confidencial, nos interesaba por ser un reflejo de un proceso que se podía generalizar a otras familias, si bien ellos eran los interlocutores con los que trabajábamos. 
Todas las sesiones fueron grabadas en una cinta magnetofónica, puesto que contábamos con la aprobación de los participantes. Con posterioridad, estas cintas fueron transcritas en papel. Asimismo, y de forma complementaria, los entrevistadores emitían un informe que recogía sus impresiones sobre el desarrollo de la sesión.

\subsection{Procedimiento de análisis de datos}

El trabajo con grupos de discusión requiere el uso de una aproximación analítica, propia de una metodología cualitativa y que se basa en el análisis de contenido (Valles, 1997). Con este propósito, se establecieron tres niveles de análisis:

1. Clasificación temática de las distintas ideas que son verbalizadas en los grupos de discusión, elaborando, a partir de ello, un sistema de categorías.

2. Descripción del contenido de las transcripciones: inclusión de comentarios de los participantes en relación con los tópicos específicos que se tratan en cada momento. Estos comentarios fueron elegidos por la claridad en la exposición de las ideas y por su significatividad y pertinencia en relación con el tópico expresado.

3. Interpretación teórica del contenido descrito en el segundo nivel de análisis. Se elaboraron conclusiones en relación con los objetivos de la investigación y tomando como puntos de referencia los desarrollos teóricos efectuados, los resultados de investigaciones similares, etc.

\section{Resultados obtenidos: los procesos de acuerdo y negociación en el reparto de las tareas domésticas}

\section{1. ¿Cómo hacemos para negociar el reparto?}

Los discursos de las parejas participantes en los grupos de discusión indican que los acuerdos sobre el reparto del trabajo doméstico son soluciones flexibles y prácticas que permiten resolver los problemas que se presentan en el día a día. Asimismo, muchas de las parejas afirman que no invierten mucho tiempo dialogando acerca de cómo ha de ser este reparto, por lo que no suele haber procesos de negociación en los que se usen reglas y procedimientos explícitos y elaborados (véase Coltrane, 1996). Así, es frecuente que se manifiesten del modo que sigue:

¿Pero eso se negocia o qué?... Yo creo que es lo que surge... Es un reparto. Ya casi nos ponemos... No sé. Por poner un ejemplo, llega la noche y con las crías, «¿voy bañándoles yo y haces tú la cena?», «Venga, vale»... (Varón, minero, $42, \mathrm{GD} 2)^{1}$

1. Cuando se inserten citas textuales de los discursos de los participantes, se incluirá, entre paréntesis, una anotación que contenga el género de quien realizó la afirmación, su profesión, edad y el grupo de discusión en el que participó. Queremos aclarar que los textos 
En nuestro caso, es que creo que no tenemos nada repartido, se llega a casa, hay que hacer esto y quien está, lo hace... Sí, hay labores que hago prácticamente al cien por cien yo y otras que hace J., porque se nos da mejor o porque empezamos así desde el principio. (Mujer, telefonista, 41)

\section{Su marido lo corrobora:}

No es que tengamos establecido esto lo tienes que hacer tú y esto lo tengo que hacer yo. (Varón, técnico de laboratorio, 44, GD1)

Las parejas no negocian explícitamente los términos del reparto. En este sentido, traemos a colación una reflexión muy oportuna que se realizó en uno de los grupos de discusión. Así, un varón establece que cuando estudiaba la carrera universitaria compartía piso con hombres y mujeres. En este contexto, la división del trabajo doméstico se negoció explícitamente, de modo que cada uno de los integrantes de la casa sabía, con claridad, las tareas que debía realizar. A partir de esta experiencia, su mujer, presente en el grupo de discusión, reflexiona sobre la conveniencia de extrapolar el sistema de negociación y reparto que se produce en estos casos al contexto de la vida familiar, si bien también reconoce la dificultad para lograrlo. Sobre este aspecto, también se pronuncia Callejo Gallego (2005), quien señala que la convivencia familiar no es fácil, pero que el proceso de negociación se puede abordar a partir del establecimiento de reglas y la búsqueda de consenso:

Otras veces vivimos a lo mejor tres mujeres y tres hombres sin relaciones digamos «afectivas», compartiendo piso, y las tareas se repartían sin ninguna duda. (Varón, funcionario, 43, GD9)

Y su esposa dice:

Pero es distinto. (Mujer, técnica de telecentro, 38, GD9)

Y su marido la contradice afirmando que:

No es distinto. Le toca el baño a Pepe, el martes le toca el baño a Luisa. Y a ver, organizabas compras y lo hacía todo dios... No había diferencia por sexos o por género dentro de la planificación de aquella casa, o sea que cuando ocurre lo contrario, son rollos que están por encima del hombre y de la mujer. $\mathrm{Ye}^{2}$ rollo cultural, tradicional.

pueden leerse desde enfoques distintos. Por la temática del artículo, los leemos para analizar los procesos de negociación en la división sexual del trabajo doméstico. Otra lectura posible es la que se centra en el estudio de las concepciones implícitas de la masculinidad y la feminidad hegemónicas que se reflejan en los discursos, lo que nos llevaría a elaborar otro artículo.

2. Se mantienen los modismos del habla local, asturiano o bable. 
Y su mujer responde:

Yo es lo que vi en vivir en pisos compartidos con otros y luego en pareja, y son situaciones distintas. Probablemente haya muchas cosas buenas de los pisos compartidos que se deberían de trasladar. Lo primero porque se establecen unas normas muy claras, y aquí vas, te casas o te vas a vivir juntos $y$, de mano, nadie pone normas, y salga el sol por Antequera, que eso es un error probablemente. En los pisos, estaba todo organizado y distribuido, y cuando alguien no cumplía, se le recriminaba o se le llamaba la atención.

Para poder entender esta dinámica discursiva, resulta útil diferenciar la estructura de las relaciones que sostienen ambos tipos de grupos, el familiar y el de amistad. Tomando como referencia las teorías del intercambio social, debemos distinguir entre las relaciones comunales y las de intercambio (Clark y Mills, 1979, citados en Grote y Clark, 1998: 244). En las primeras, sus miembros se sienten responsables del bienestar de los otros y la ayuda prestada se proporciona sin esperar nada a cambio, por lo que no se crea la necesidad de devolver el favor recibido. Por otra parte, en las relaciones de intercambio, las personas dan algo para recibir otra cosa en contrapartida, de modo que lo que reciben ha de ser similar a lo que proporcionaron. En este tipo de relaciones, el favor que se da crea un deber.

Pues bien, las relaciones que se establecen entre las personas que comparten un piso son, por lo general, de intercambio. Por ello, es lógico suponer que haya unas reglas, claramente definidas y compartidas por todos, que concretan los procesos de distribución de las tareas domésticas y cuyo incumplimiento puede provocar la aparición de conflictos. Por el contrario, las relaciones familiares son relaciones comunales y, en ellas, se presta menos atención a la definición, clara y explícita, de los deberes de cada uno de los miembros de la unidad familiar. La negociación sobre el reparto se diluye en un proceso informal, en el que la decisión cartesiana de dividir las tareas al cincuenta por ciento puede verbalizarse, pero pocas veces se traslada a la práctica.

Asimismo, cuando las parejas reconocen que negocian explícitamente determinados acuerdos sobre el reparto observamos, ateniéndonos nuevamente a la lógica de las relaciones comunales, que el proceso se conduce en base a la necesidad de ajustar prioridades y situaciones personales. La negociación se realiza a partir de la búsqueda de la igualdad y la justicia distributiva, lo que provoca que no todos los miembros del grupo familiar se impliquen de igual forma en las tareas domésticas:

Tenemos establecidas unas prioridades. Yo colaboro mínimamente, pero trabajo sesenta horas a la semana. Tenemos establecida la prioridad de que descanso en cuanto a las tareas de la casa. (Varón, construcción, 38, GD4)

Cuando nos casamos, hablamos y, bueno, mientras yo esté en casa, yo asumo el trabajo de la casa, porque me parece injusto que, después de estar ocho horas trabajando, tengas que llegar a casa y colaborar con las labores de la casa, pero 
en el momento en que yo empiece a trabajar, también es justo que repartamos las labores de la casa. (Mujer, ingeniera, 36, GD8)

Asimismo, y en consonancia con la dinámica propia que rige las relaciones comunales, muchas de las parejas son conscientes del hecho que la convivencia supone un proceso de "ceder y recibir», lo cual exige la reevaluación constante de los esquemas personales para hacer la vida en pareja menos conflictiva. Y es que, a consecuencia de los procesos de socialización divergentes en los que los cónyuges han estado inmersos, cada uno acude a la relación con una visión particular de cómo deben ser hechas las tareas de casa y de cómo debe enfrentarse el proceso de vivir en pareja.

Yo no sé si coincide que en la casa en la que vivimos primero estaba yo sola y luego vino a vivir conmigo y al principio hubo incluso bastantes roces, pues porque yo tenía mis cosas, y yo quería más o menos que aquello estuviera a mi manera. Me costó trabajo aceptarlo, pero aprendí a hacerlo. (Mujer, profesora, 43, GD1)

\section{Y su marido corrobora más adelante:}

Yo recuerdo las primeras riñas en convivencia. Había una cosa que me espantaba, ella es la típica persona que dice: «Tengo casa, hay que poner lo que se necesita, que me lo solucionen rápido, y no piensa más». Y yo soy de una mentalidad totalmente diferente, a mi me gusta sentirme identificado con las cosas de la casa, entonces no me importa que la sala esté vacía, ya llegará a su debido tiempo. Yo recuerdo que la primera vez que discutimos fue un día que $\mathrm{M}$. fue a un establecimiento y dijo: "Yo necesito", y me llegó con unos cuadros horrendos porque una pared estaba desnuda y no podía quedar sin nada. Yo me sentí muy mal y lo dije. (Varón, monitor medioambiental, 45, GD1)

Este proceso de mutuo acoplamiento también se observa cuando algunas parejas constatan que la participación masculina en el hogar ha aumentado con el paso de los años. Algunas mujeres reconocen que sus maridos han sido socializados en base a un modelo tradicional y que han intentado funcionar con la lógica de este modelo. Ello derivó en situaciones conflictivas que condujeron, progresivamente, a una mayor participación del varón:

Yo antes en casa no hacía nada. Cuando empezamos a vivir juntos, yo, al salir de la mina, no hacía más nada, y poco a poco hay que ir cambiando el chip y no haré todas las cosas que ella quiere todavía, pero muchas más de las que hacía, sí. (Varón, prejubilado, 45, GD5)

Pero sí me doy cuenta que a lo mejor a él, como a muchos otros hombres, no lo enseñaron a lavar los baños, por ejemplo... He tratado de irlo enseñando, porque me parece lógico que, si vamos a compartir las tareas, pues se las enseñe si no las sabe hacer. (Mujer, ingeniera, 36, GD8) 
Para llegar a la situación en que estamos ahora mismo tardamos un año y tuvimos que cambiar el sistema tradicional que traíamos de detrás. Yo tengo cuatro hermanas y mi madre. Yo estaba muy mimado. Que yo me acuerde, mientras estuve soltero, nunca hice ni una sola tarea de casa. (Varón, prejubilado, 46, GD9)

\subsection{Las fuentes del conflicto sobre el reparto del trabajo familiar}

En el proceso de vida en común, las situaciones derivadas de la necesidad de repartir las tareas domésticas pueden ser vistas desde diferentes perspectivas por parte de los integrantes del grupo familiar. Esta situación puede acarrear la aparición de conflictos. A continuación, exponemos algunas de las fuentes del conflicto sobre el reparto de las tareas.

\subsubsection{El conflicto provocado por la escasa participación masculina en las tareas domésticas}

La mayoría de las parejas reconocen la existencia de conflictos cuando negocian el reparto de las tareas. En nuestro caso, hemos visto que la principal fuente de conflicto deriva de la exigencia femenina de que su pareja participe más (véase también Coltrane, 1996; Deutsch, 1999; Kluwer, 1998; Tobío, 1998). Los hombres han sido los principales beneficiados con el reparto de tareas domésticas, por lo que están menos dispuestos a demandar cambios; mientras que las mujeres no tienen nada que perder y mucho que ganar si consiguen transformar la dinámica familiar. Asimismo, en los siguientes textos, también observamos un supuesto que sigue funcionando: si la mujer es ama de casa, todo el trabajo doméstico es para ella, no importa el tiempo que ocupa, ni tampoco entiende de vacaciones o fiestas:

Me da mucha rabia, porque yo no trabajo fuera de casa, pero al final yo estoy muchas más horas trabajando, porque yo me levanto cuando J., pero yo a las doce de la noche sigo trabajando. Eso tampoco es justo. (Mujer, ama de casa, 38, GD3)

Referente a la convivencia que tuve, hay dos frases que siempre me quedaron de él [se refiere a su ex marido]. Cuando yo le pedía si me echaba una mano, decía: «¿Tiene que ser ahora?». La frase favorita de él: «¿Tiene que ser ahora?», «Hombre, si te parece que lo dejemos para mañana...». O cuando decía: «¿Dónde está esto?, ¿Dónde está el trapo?», entonces terminas respondiendo: «Trae, anda, que lo hago yo», porque tardas más tiempo en ponerle las cosas ahí delante que... (Mujer, auxiliar administrativa, 43, GD8)

Las tareas de casa las hago yo todas. Y si algún día tiene que hacer algo, ayer, por ejemplo, fregó los cacharros de noche, pero yo creo que era porque teníamos que venir a esto [se refiere al grupo de discusión], pero lo hago yo todo. (Mujer, ama de casa, 35, GD5) 
Yo lo único que me duele y me molesta mucho de ti [se está dirigiendo a su marido] es que, aun pudiendo, no te dediques más al cuidado de las crías... (Mujer, ama de casa, 39, GD4)

Igualmente, algunas mujeres, cansadas de no encontrar una respuesta satisfactoria en sus maridos, confiesan que han optado por dejar de insistir. Reconocen su fracaso y evitan las discusiones, al creer que no les llevarán a ninguna parte. Se produce un «diálogo de sordos» que evita la posibilidad de llegar a acuerdos negociados, lo que provoca una profunda insatisfacción en la mujer:

Me cuenta él su película y yo digo que, por no discutir, se acabó el tema... Simplemente, decir, pues, una tarde: «¡Anda bánalos tú, les preparas la merienda y les cuentas tú el cuento!», y te contesta no sé que, no sé cuánto. En ese sentido, las mujeres, por no discutir, decimos: «Anda, venga, que lo hago yo». (Mujer, negocio propio, 44, GD3)

Es interesante analizar la dinámica de pareja que se refleja en el fragmento discursivo. Ante los requerimientos de la mujer para que su marido se encargue de las tareas de cuidado, se muestra reticente y las evita con disculpas que ella menciona bajo la expresión "no sé que, no sé cuánto». Ante esta situación, la mujer, ya exasperada, asume todo el trabajo. Observamos un proceso comunicativo en el que él intenta evitar el trabajo y ella refuerza, inconscientemente, esta conducta. Ella asume todo el trabajo, se enfada y se produce el conflicto.

\subsubsection{El conflicto provocado por la coexistencia de dos formas distintas de realizar las tareas domésticas}

Otro importante foco de discusión tiene que ver con los diferentes estilos y maneras en que hombres y mujeres abordan la realización de las tareas de la casa. En estos casos, la mujer es la que las organiza y el resto de miembros de la familia ha de acomodar su participación para alcanzar los niveles requeridos por ella. Muchas mujeres y hombres constatan que las mujeres son más exigentes y perfeccionistas. Esto es motivo de discusión, pues muchos hombres constatan que, para sus mujeres, la casa nunca está limpia del todo. Estas ideas están en consonancia con las conclusiones que Tobío (2005) obtiene de su investigación, al constatar que un aspecto en el que parece que la mujer es insustituible es en el establecimiento y el mantenimiento del «orden» en el hogar. Es como si el orden y la limpieza proporcionasen una sensación de tenerlo todo bajo control. En algunos casos, esa necesidad se convierte en una obsesión que puede ser fuente de conflictos:

Yo alguna vez fui maniática de la limpieza, pero la realidad te va enseñando. Llega un momento que dices: «Estoy perdiendo el tiempo limpiando. Al final se va a ensuciar otra vez y vas a tener que volver a limpiar». (Mujer, ingeniera, 36, GD8) 
Me gustaría saber cómo hacéis [pregunta al resto de mujeres del grupo] para poder pasar del polvo y de la limpieza de casa... Es que no puedo pasar. (Mujer, ama de casa, 42, GD5)

Y su marido replica:

Es que ta todo el día: limpia, limpia. (Varón, prejubilado, 45, GD5)

Y ella se justifica diciendo que:

Es que es una casa grande y yo tengo que limpiarla todos los días y verla limpia, y yo lo intento, porque a veces me dice P.: «Pero pasa, deja un poco», y es superior a mí. O sea, yo salir a la puerta, mirar atrás y ver que queda sucio es imposible.

Si se analizan los dos primeros textos, podemos contrastar, con claridad, el imaginario relativo a la limpieza que posee un ama de casa y el que asume una mujer que trabaja fuera del hogar. En los dos casos, ambas relatan su obsesión por la limpieza; sin embargo, en el segundo texto, la centralidad del trabajo doméstico provoca una satisfacción con el «trabajo bien hecho»; además, si no lo hiciese de este modo, el trabajo perdería todo su sentido. En cambio, en el primer texto, se constata la evolución vital hacia una situación en la que se relativiza el nivel de limpieza, quizás a consecuencia de las mayores dificultades para conciliar ambos mundos y a que la esfera doméstica no ocupa un lugar tan destacado en su cotidianeidad.

A su vez, también se constata que a las mujeres les gusta el orden, por lo que las tareas se hacen con prontitud y celeridad. Se concluye que hombres y mujeres tienen distintas prioridades y distintos ritmos para hacer las faenas de casa. Es más, es frecuente que las mujeres que se dedican en exclusiva a las tareas del hogar tengan jornadas laborales más amplias que sus maridos, quizás porque siempre encuentran algún trabajo que realizar. También es muy frecuente que las diferencias de género se achaquen a distintas disposiciones personales: ellas tienen más «nervio» y ellos son más «tranquilos». En este contexto, esa diferencia de esquemas puede provocar conflictos familiares, pues la mujer desea que las tareas se hagan de una determinada manera, mientras que los hombres no asumen los patrones de orden y organización establecidos por ella:

Yo también plancho, pero yo puedo ver la ropa amontonada. Ella no puede ver ropa amontonada. (Varón, técnico de laboratorio, 44, GD1)

Yo a veces estoy cansadísima y le estoy diciendo a J.: «Hoy no plancho!». Me pego un par de vueltas por ahí, me salgo afuera a la calle, como vivimos en el campo, y, bueno, enchufo la plancha y me gusta, porque me he quitado una cosa que no la voy a tener que hacer al día siguiente... La forma de ser, que somos muy diferentes J. y yo. (Mujer, ama de casa, 38, GD3) 
Tú ves y tienes algo para planchar y empiezas a sudar hasta en verde. Ellos, mientras que haya camisas en el armario... (Mujer, funcionaria, 37, GD1)

A lo mejor tenía catorce camisas porque sólo planchaba una vez a la semana o cada quince días. O sólo lavaba una vez a la semana o cada quince días, entonces tenía que tener veinte pares de camisas... Y, vosotras las mujeres, esa minuciosidad y la tenacidad en el trabajo. Yo creo que parte directamente del aprendizaje tradicional de casa. (Varón, funcionario, 43, GD9)

Asimismo, en los discursos anteriores, se observa la primacía de viejos elementos constitutivos del ethos femenino del patriarcado, que reflejan y atribuyen a la mujer un estatus dependiente y subsidiario respecto al varón. Así, la mujer «debe» tener el tiempo siempre ocupado, incluso el ocio, para no perder la virtud y el sentido de la pureza moral que se refleja en el aspecto externo de las cosas: «La casa es el espejo del alma» (Bourdieu, 2000).

Además, muchas mujeres reconocen que tardan menos tiempo que sus maridos en hacer las labores domésticas. Como consecuencia del vertiginoso ritmo de vida actual, esta actitud puede provocar la reproducción de los estereotipos de género, pues se concluye que, puesto que la mujer lo hace más rápido y mejor, es más conveniente para la familia que lo haga ella. Así, y como veremos más adelante, es frecuente que muchos hombres justifiquen su escasa participación señalando que sus mujeres lo hacen mejor y que ellos son unos inútiles (véase también Bianchi, Milkie, Sayer y Robinson, 2000; Coltrane, 1996).

\subsubsection{El conflicto ocasionado por las dificultades de las mujeres para delegar las tareas domésticas}

Una fuente importante de conflicto familiar deriva de las dificultades que tienen las mujeres para delegar la realización de las tareas domésticas. Las mujeres están constantemente pendientes de todos los asuntos familiares y constatan su dificultad para compartir. Se produce una situación paradójica, pues, por un lado, se quejan de que los varones participan poco, pero, al mismo tiempo, son reacias a perder su parcela de poder doméstico (véase también Alberdi y Escario, 2007; Deutsch, 1999; Doucet, 2001; Tobío, 2005; Torns, Borrás y Carrasquer, 2003-2004). En este sentido, algunas de las mujeres entrevistadas por Tobío (2005) reconocen que suelen tener ideas bastante rígidas acerca de cómo se deben hacer los trabajos domésticos y tienden a imponerlas.

En nuestros grupos de discusión, se comentó que las mujeres ejercen una férrea vigilancia de la conducta del varón y que, si observan que éste no hace las tareas como creen que deben ser hechas, las realizan ellas, con lo cual muestran su incapacidad para delegar:

Estoy haciendo cualquier cosa y viene ella a decirme: «Esto no se hace así». La costumbre en mi casa es que el último que se levanta, hace la cama, y cuando yo la hacía, ella retocaba, y entonces dije: «Ahora no hago la cama. Total, vas a hacerla tú después». (Varón, prejubilado, 48, GD2) 
Vamos a poner un ejemplo. Pones el aceite, te pones a freír las patatas y ella está por la cocina, pero ella está con un ojo en el cazo y el otro en lo que está haciendo. Echas una patata: «iNo la eches, que no está caliente todavía!» o «¡Ves, ahora sale mucho humo. Está muy caliente!». (Varón, mecánico, 39, GD6)

Me parece que haciéndolo yo, que acabo antes, y no sé si es que lo hace y me parece que no quedó bien, entonces tengo que ir yo detrás. Prefiero hacerlo yo. (Mujer, ama de casa, 35, GD5)

Asimismo, algunos hombres, aunque no todos, se quejan de que las mujeres no saben delegar las tareas de cuidado infantil. Alberdi y Escario (2007) indican que algunos de los participantes en su estudio señalan que sus mujeres ponen barreras a su participación en las labores de crianza infantil. Estas autoras afirman que algunas mujeres saben que, para incorporar a los varones, tendrán que retirarse a un plano secundario y perder algo del poder que ostentan en la esfera doméstica. Sobre el particular, algunas personas de nuestra investigación constatan que:

Nos cuesta muchísimo delegar con un bebé recién nacido, ¿a qué sí, R.? Ahí sí lo viviste e incluso me lo recriminó en algún momento. (Mujer, técnica de telecentro, 38, GD9)

Yo los diez primeros días lo bañé, lo limpié y yo creo que ella se celaba. Ella no se encontraba al principio bien, y tenía ganas de empezar a bañarle y... (Varón, guarda forestal, 45, GD9)

Es que le delego, pero explicándole. Una vez que fue a urgencias, le dije: «El niño tiene esto, tomó esto». Le apunté en un papel. Él se puede acordar, pero a lo mejor no se acuerda. Y veo que le pongo la cabeza hasta aquí, pero yo se lo tengo que decir. (Mujer, ama de casa, 38, GD3)

Los estudios corroboran que la actitud de las madres ante la participación del padre en el cuidado infantil ejerce una influencia poderosa que favorece o entorpece los esfuerzos paternales (Allen y Hawkins, 1999; Bulanda, 2004; Fagan y Barnett, 2003). Estos autores han mostrado que muchas mujeres se resisten a perder el rol principal en el cuidado infantil, pues consideran que ésta es su responsabilidad y creen que sus parejas masculinas no disponen de las habilidades necesarias para desempeñar dicho rol con eficacia. Esta situación puede actuar como barrera que impide la mayor participación paternal.

Las tres fuentes principales de conflicto que hemos relatado pueden converger en un mismo proceso de negociación y discusión marital. Cada uno de los dos miembros de la pareja acude a la negociación con ideas, prejuicios, esquematismos, etc., que definen cual ha de ser la posición de cada uno respecto a la corresponsabilidad familiar. Por tanto, en la negociación sobre la división del trabajo doméstico, pueden converger dos maneras distintas de 
entender cómo deben hacerse las cosas de casa, y ese choque de cosmovisiones provoca la aparición de conflictos. En unos casos, estos conflictos tienen su origen en alguna de las cuestiones que acabamos de definir; sin embargo, en la mayoría de los casos, pueden converger todos o casi todos los temas descritos en el presente epígrafe.

\subsection{Estrategias masculinas para evitar el trabajo doméstico}

En ocasiones, se produce una «resistencia pasiva», en palabras de Deutsch (1999), de modo que algunos hombres dan la callada por respuesta y tratan de acomodarse a la situación de privilegio que ostentan. Ante la petición de participación por parte de sus parejas, optan por ignorarla. En ocasiones, los varones reconocen el uso de esta estrategia y usan distintos argumentos para justificar las razones que los impelen a actuar de este modo:

No me desagrada cocinar, pero reconozco que, si puedo, me escurro. (Varón, técnico de laboratorio, 44, GD1)

Cuando llego a casa, me gusta más relajarme que ponerme a hacer cualquier otra cosa. No me cuesta ningún trabajo si algún día hay que fregar los cacharros, pero no participo mucho, la verdad es que más bien poco, tirando muchas veces a nada. (Varón, maderista, 42, GD7)

También hay veces, cuando está cansado, que hace lo de callar. Cuando no quiere hacer algo, se calla o se hace el sordo... (Mujer, ama de casa, 38, GD3)

Ante esta argumentación, su marido cuenta:

Trabajo fuera y llego a casa y no puedo estar tranquilo media hora y, a veces, no es escurrir el bulto, es un poco de higiene psicológica. Yo por eso a veces salgo. A lo mejor, ella dice que es escurrir el bulto. (Varón, profesor, 44, GD3)

\section{Y su mujer contraataca:}

Es que yo también lo necesito... A mí me encantaría hacer lo que hace él en el prao, pero como yo ya he dicho que lo primero son los niños, entonces si veo que él escurre el bulto, porque le apetece o necesita salirse fuera, me quedo yo dentro, me fastidio. Y es que se lo digo: «¿Por qué no te quedas tú dentro y salgo yo?». Es que lo necesito también.

Es interesante analizar el intercambio discursivo en esta última pareja. Él reconoce que, a veces, llega a casa cansado y que necesita salir al prado para respirar, para descansar. En consecuencia, cree que no está escurriendo el bulto. Ante esto, su mujer comenta que ella también necesita cierto respiro. Podemos concluir que ambos necesitan tiempo para ellos mismos, pero que, en el caso de la mujer, las obligaciones de cuidado infantil le impiden disfrutar de ese 
tiempo libre. En este contexto, ella considera que lo que hace su marido es «escurrir el bulto». Observamos que el mismo hecho es analizado desde dos cosmovisiones distintas: la de la mujer, que prioriza la atención y el cuidado a sus hijos por encima de sus propias necesidades psicológicas, y la de su marido, quien encuentra siempre algo de tiempo para él mismo. Nos percatamos que no ponen en común las distintas cosmovisiones rutinizadas de las que parten, de modo que son incapaces de ponerlas en entredicho y negociar un reparto que satisfaga a ambos.

En otros casos, se establece que los hombres se van acomodando a la situación cotidiana que les ha tocado vivir, de modo que, en vez de participar cada vez más en las labores domésticas, se produce la situación contraria. En este contexto, es interesante volver a resaltar la dinámica interactiva que se establece entre las parejas cuando se produce tal situación, pues hemos constatado que, la mayoría de las veces, se originan conflictos porque las féminas consideran injusta la situación que se ha creado:

En tu caso [se refiere a su marido], tú te has acomodado porque es muy fácil acomodarse. Seguramente, yo si fuera hombre también me acomodaría. (Mujer, ama de casa, 39, GD4)

Creo que, con los años, nos vamos haciendo como más cómodos, porque yo antes hacía muchas más cosas en casa de lo que hago ahora. (Varón, guía turístico, 40, GD4)

Había un reparto muy equitativo [se refiere a los primeros años de su matrimonio] y, a lo largo del tiempo, veo que el reparto está más descompensado. Yo tengo más y él, algo menos, pero también es algo que no se ha hablado. Yo voy cogiendo por naturaleza, él va dejando por situación... (Mujer, dirección de servicios sociales, 42, GD8)

\section{Y su marido corrobora esta visión:}

A lo largo de estos años, fui perdiendo tensión en ese tipo de cosas... También reconozco que la capacidad organizativa de ella es mucho mejor que la mía y entonces me dejo llevar. (Varón, analista de sistemas, 45, GD8)

El último intercambio discursivo resulta sumamente interesante, en la medida que nos muestra el proceso paulatino hacia una división sexual del trabajo doméstico en una pareja de doble ingreso que lleva varios años casada. En este caso, la vida matrimonial no evoluciona hacia un reparto más equitativo, sino que ocurre la situación inversa. Resulta muy sugestiva la frase pronunciada por la mujer, quien señala que: «Yo voy cogiendo por naturaleza, él va dejando por situación». Parece sugerir una naturaleza femenina - feminidad hegemónica- que adopta las tareas de mando y organización doméstica; mientras que, a ella, se contrapone una naturaleza masculina — masculinidad hegemónicaque hace menos labores del hogar con el paso del tiempo. Se reafirma la vieja 
idea del ethos femenino, en donde la mujer, dada su "naturaleza», es relegada y ocultada al ámbito de la casa. En este caso, el varón también justifica su menor participación reconociendo las mayores y mejores habilidades domésticas de su mujer y su propia tendencia a evitar estas tareas.

Enlazando con la idea anterior, algunos hombres reconocen que no tienen que hacer las tareas domésticas porque no saben y nadie les ha enseñado a hacerlas. A su vez, refuerzan esta argumentación constatando que a su mujer se le dan muy bien estas labores. En suma, se escudan en la máxima de que: «Yo no sé hacerlo y ella lo hace mejor». La falta de habilidad doméstica masculina y la gran capacidad femenina para emprender este tipo de tareas se aúnan para justificar la menor implicación masculina:

De la casa, no sabe nada. Tampoco se molesta en aprender, porque le digo: «Pues aprende, jobar! ¡Aunque sea un día, haz una cena, haz algo!». Él responde: «Tú haces esas cosas porque se te dan mejor». Digo: «Pero, ¿por qué se me dan mejor? Cuando yo tuve a la niña, no sabía lo que era un pañal. No tenía a mi madre ni a nadie que me enseñara. ¿Tú te crees que yo lo sabía? No lo sabía. Lo mismo que lo aprendí yo, podías haberlo aprendido tú». (Mujer, negocio propio, 44, GD3)

Cuando la cría tenía tres años, me vi haciendo una coleta por primera vez en mi vida. Imaginaros. Claro, dices: «Si está la madre, que lo haga la madre, que lleva haciendo coletas toda la vida». En muchos aspectos logísticos y de organización es muy buena, y entonces esa parte la delegas, porque funciona muy bien. (Varón, analista de sistemas, 45, GD8)

El último fragmento constata la conclusión de Deutsch (1999), quien indica que los hombres suelen combinar un discurso que enfatiza su falta de habilidad con la alabanza a su mujer. Pero si bien la alabanza puede ser una expresión sincera de aprecio y afecto, también es una forma de mantener el trabajo doméstico como un dominio femenino. Para esta autora, la alabanza es un arma de doble filo; por una parte, hace que las mujeres se sientan apreciadas y reconocidas en su trabajo doméstico; pero, por otra, puede socavar la lucha de la mujer para conseguir más ayuda, al no querer perder los sentimientos de autoestima positiva derivados de la admiración que provoca en su pareja.

Asimismo, con mucha frecuencia, este tipo de discurso enmascara la poca disposición masculina a aprender, constatando que «los hombres no saben y, en principio, no quieren aprender» (Torns, Borrás y Carrasquer 2003-2004: 125). Deutsch (1999) indica que los hombres pueden argumentar que su incompetencia no es una estrategia, sino que refleja su poca pericia para realizar trabajos que no saben hacer. Esta autora se opone a esta argumentación por dos cuestiones fundamentales: en primer lugar, indica que, si bien las mujeres han sido socializadas para dedicarse intensamente a las labores del hogar, la diferencia principal entre hombres y mujeres estriba en que ellas saben que las tareas "deben" hacerse, no hay posibilidad de elección. Además, Deutsch señala que las rutinas del hogar pueden aprenderse rápidamente. En consecuen- 
cia, señala que el meollo de la cuestión no está en la falta de competencia, sino en la escasa motivación para afrontar la realización de estas labores.

Igualmente, algunos hombres constatan que, después de trabajar todo el día fuera de casa, se encuentran muy cansados para hacer las tareas domésticas o para participar en las labores de cuidado de niños y niñas. Es paradójico que estos varones no asuman que sus mujeres también trabajan fuera de casa, están cansadas y, sin embargo, también realizan labores domésticas.

Me cuenta su película, que él está fuera, que el estar en casa es estar mucho más tranquilo, que él tiene que pelear con una empleada, con otra, con el proveedor, con la clienta que entró y no sé cuántos... Y dices tú: «Bueno, bueno...». Pero, bueno, al fin y al cabo es trabajo y los dos estamos trabajando. Y yo tampoco es que le pida mucho. (Mujer, negocio propio, 44, GD3)

A su vez, en algunas ocasiones, los procesos de discusión que se originan implican un proceso de comparación de la participación de ambos miembros de la pareja con respecto a otras parejas de su entorno familiar y social. En estos casos, algunos hombres recurren a la estrategia de justificar su menor participación indicando que colaboran en el hogar tanto o más que otros varones de su entorno (véase también Deutsch, 1999). En otras ocasiones, este proceso de comparación se establece con generaciones anteriores:

Yo creo que hago mucho más, muchísimo más, de lo que hacía mi padre. ¿Que no hago lo que puede hacer un chaval de veinticinco? Tampoco es que ellos hagan mucho. Habría que verlo. (Varón, comercial, 48, GD4)

En algunas ocasiones, las mujeres reconocen que sus maridos sobreestiman su participación en la realización de las tareas domésticas. Ello corrobora las conclusiones de Kamo (2000), quien ha constatado que las discrepancias entre lo que se hace y lo que se dice que se hace son mayores en los varones. Asimismo, debemos recordar aquí las conclusiones del estudio de Coltrane (1996), quien constata que las actividades que se realizan esporádicamente tienden a retenerse en la memoria, de modo que, si una tarea se hace de forma esporádica, hay una tendencia a recordar cada ocasión en la que se ha hecho. Coltrane pone el ejemplo de un hombre que hace pocas veces la comida. Dado que es un evento ocasional, considera que éste será recordado de forma más vívida y, por ello, tenderá a sobreestimarse. Desde esta perspectiva, Coltrane señala que muchas de las mujeres a las que entrevistó para su investigación indicaban que sus maridos recordaban cada pequeña contribución que habían realizado a las tareas domésticas:

Y dice [se refiere a lo que dice su marido]: «Aquella vez, hace tres años, ¿te acuerdas, que me tocó hacer y madrugué toda una semana seguida, apenas dormí y apenas comí porque tuve que estar...?», pues ya libramos el resto del año, no del año, de todos los años venideros. (Mujer, negocio propio, 38, GD3) 
Por último, debemos mencionar que, de forma excepcional, algunos varones no participan en las tareas del hogar porque sienten que no es su trabajo. Sobre el particular, también se pronuncia Tobío (1998), quien, en su estudio, concluye que, cuando los hombres realizan labores domésticas, subyace la idea de excepcionalidad o de asunción de una tarea que, en realidad, corresponde a las féminas. Así, por ejemplo, se dice que:

Pues alguna vez me contestó que es mi trabajo y que lo tengo que hacer. (Mujer, ama de casa, 35, GD5)

Pero, vamos a ver, yo estuve veinte años metido en la mina y nadie me ayudó. Yo tenía que hacer lo mío. Ahora creo que respiré y no participo. Antes tampoco participaba. (Varón, prejubilado, 43, GD2)

Sin lugar a dudas, detrás de estas afirmaciones, se aprecia la idea de que el trabajo del hogar es una labor femenina. En el último fragmento, el varón asume una división sexual del trabajo: en su caso, él trabajaba en la mina y su mujer, en casa. En lógica consecuencia con este hecho, asume que las cosas no tienen por qué cambiar, aunque su situación laboral haya sufrido una transformación.

\section{Discusión de los resultados}

Si bien la experiencia del trabajo doméstico ya no es ajena a los hombres, la fuerza de los convencionalismos de género todavía se deja sentir. Hombres y mujeres realizan tareas domésticas, pero la forma en que éstas se negocian refleja la persistencia del dualismo de género. Debido a la fuerza de los estereotipos, el trabajo doméstico tiene para unos y otras un significado totalmente distinto que condiciona la negociación sobre el reparto. Así, las discusiones y los conflictos generados manifiestan esa diferencia de enfoques y perspectivas, que se puede resumir en que para las mujeres las responsabilidades domésticas son prioritarias, mientras que los varones, aunque participen, son conscientes, y lo asumen, de que desempeñan un papel secundario.

Ellos se quejan de que a sus mujeres les cuesta delegar y de que son muy exigentes en cuanto al nivel de desempeño doméstico. Ellas, sin embargo, expresan su disconformidad por la escasa participación de sus parejas. En resumen, distintas cosmovisiones que son el caldo de cultivo en el que se gestan la negociación y el conflicto en torno al proceso de división del trabajo familiar. Dichas cosmovisiones rutinizadas e interiorizadas afloran en las discusiones que surgen en los grupos y no se ponen en entredicho, por lo que no se gestan procesos explícitos y formales de negociación.

Ante esta situación, finalizamos el artículo cuestionándonos la posibilidad que se produzca un cambio en los hogares. ¿Es esto posible?, ¿bajo qué circunstancias y condiciones se puede mejorar la negociación sobre el reparto? Queremos sugerir la conveniencia y la necesidad de implementar programas 
de educación para la vida familiar. Así, si bien en otros países del entorno anglosajón son abundantes la iniciativas de este tipo, en el nuestro son escasos. Estos programas deben favorecer y promover actitudes más favorables hacia el reparto equitativo de las tareas domésticas y ayudar a las parejas a establecer cauces para negociar y gestionar ese reparto (Apparala, Reifman y Munich, 2003; Bartau, Maganto y Etxeberría, 2002; McBride y Lutz, 2004). A través de la intervención, es necesario ayudarles a que exterioricen su esquema de acción y que sean conscientes de éste, permitiendo que reflexionen sobre cómo se ven a sí mismos y a sus parejas. Asimismo, es importante el trabajo de habilidades sociales que permitan expresar sentimientos y proyectar, como espejos, el absurdo de determinadas relaciones y preconcepciones.

\section{Referencias bibliográficas}

AlBerdi, I. (1999). La nueva familia española. Barcelona: Taurus.

AlBERDI, I. y EsCARIO, P. (2007). Los hombres jóvenes y la paternidad. Bilbao: Fundación BBVA.

Allen, S. y HaWKINS, A. (1999). «Maternal gatekeeping: mothers' beliefs and behaviours that inhibit greater father involvement in family work». Journal of Marriage and the Family, 61, 199-212.

Almeida, D.; Maggs, J. y Galambos, N. (1993). «Wives' employment hours and spousal participation in family work». Journal of Family Psychology, vol. 7, 2, 233244.

Apparala, M.; Reifman, A. y MunsCh, J. (2003). "Cross-national comparison of attitudes toward fathers' and mothers' participation in household tasks and childcare». Sex Roles, vol. 48, 5/6, 189-203.

BartaU, I.; MagANTO, J. y ETXEBERRía, J. (2002). «La implicación en el trabajo familiar: fuentes de influencia e implicaciones educativas». Revista de Educación, 329, 349-371.

BaXter, J.; HewitT, B. y Western, M. (2005). «Post-familial families and the domestic division of labour». Journal of Comparative Family Studies, 583-600.

BiAnChI, S.; Milkie, M.; SAYER, L. y Robinson, J. (2000). «Is anyone doing the housework? Trends in the gender division of household labor». Social Forces, vol. 79, 1, 191-228.

Bourdieu, P. (2000). La dominación masculina. Barcelona: Anagrama.

BRULLET, C. (1996). «Prácticas de crianza e identidades parentales». En: BRULLET, C. y CARRASQUER, P. Sociología de las relaciones de género. Madrid: Ministerio de Trabajo y Asuntos Sociales, 45-65.

Bulanda, R. (2004). «Paternal involvement with children: the influence of gender ideologies». Journal of Marriage and Family, 66, 40-45.

CALlejo GALleGO, J. (2005). «Estrategias temporales: relaciones entre tiempo de trabajo remunerado y tiempo de trabajo doméstico». Cuadernos de Relaciones Laborales, $1,75-204$.

Coltrane, S. (1996). Family man: Fatherhood, housework and gender equity. Nueva York: Oxford University Press.

- (2000). «Research on household labor: modelling and measuring the social embeddedness of routine family work». Journal of Marriage and the Family, 62, 12081233 . 
Cunningham, M. (2005). «Gender in cohabitation and marriage: The influence of gender ideology on housework allocation over the life course». Journal of Family Issues, vol. 26, 8, 1037-1061.

DAVIS, S. y GREENSTEIN, T. (2004). «Cross-national variations in the division of household labor». Journal of Marriage and Family, 66, 1260-1271.

DEUTSCH, F. (1999). Halving it all. How equally shared parenting works. Cambridge, Massachusetts: Harvard University Press.

DOUCET, A. (1995). "Gender equality and gender differences in household work and parenting». Women's Studies International Forum, vol. 18, 3, 271-284.

- (2001). "You see the need perhaps more clearly than I have”. Exploring gendered processes of domestic responsibility». Journal of Family Issues, vol. 22, 3, 328-357.

FAGAN, J. y BARNETT, M. (2003). "The relationship between maternal gatekeeping, paternal competence, mothers' attitudes about the father role, and father involvement». Journal of Family Issues, vol. 24, 8, 1020-1043.

GreEnstein, T. (1996a). "Gender ideology and perceptions of the fairness of the division of household labor: effects on martial quality». Social Forces, vol. 74, 3, 10291042.

- (1996b). «Husbands' participation in domestic labor: interactive effects of wives' and husbands' gender ideologies». Journal of Marriage and the Family, 58, 585595.

Grote, N. y Clark, M. (1998). «Distributive justice norms and family work: what is perceived as ideal, what is applied, and what predicts perceived fairness?». Social Justice Research, vol. 11, 3, 243-269.

KAMO, Y. (2000). "'He said, she said": Assessing discrepancies in husbands' and wives' reports on the division of household labor». Social Science Research, 29, 459-476.

KLUWER, E. (1998). "Responses to gender inequality in the division of family work: the status quo effect». Social Justice Research, vol. 11, 3, 337-357.

KroskA, A. (2004). "Division of domestic work. Revising and expanding the theoretical explanations». Journal of Family Issues, vol. 25, 7, 900-932.

KRUEGER, R. A. (1991). El grupo de discusión: Guia práctica para la investigación aplicada. Madrid: Pirámide.

MCBRIDE, B. y LUTZ, M. (2004). «Intervention: changing the nature and extent of father involvement». En: LAMB, Michael (ed.). The role of the father in child development. New Jersey: John Wiley \& Sons, 446-475.

Meil LANDWERlin, G. (1997a). «La redefinición de la división del trabajo doméstico en la nueva familia urbana española». Revista Española de Investigaciones Sociológicas (REIS), 80, 69-93.

- (1997b). «La participación masculina en el cuidado de los hijos en la nueva familia urbana española». Papers, 53, 77-99.

Mikula, G. (1998). "Division of household labor and perceived justice: a growing field of research». Social Justice Research, vol. 11, 3, 215-241.

Navarro, L. (2006). "Modelos ideales de familia en la sociedad española». Revista Internacional de Sociología (RIS), 43, 119-138.

PAPÍ, N. y FraU, M. J. (2005). «La conciliación del empleo y del hogar: respuesta y reflejo de una organización del trabajo construida desde la institución del género». Revista Española de Investigaciones Sociológicas (REIS), 110, 149-174.

Pérez-Díaz, V.; Chulia, E. y Valiente, C. (2000). La familia española en el año 2000: Innovación y respuesta de las familias a sus condiciones económicas, politicas y culturales. Madrid: Fundación Argentaria / Visor. 
PitTMAn, J. y BlanCHARD, D. (1996). «The effects of work history and timing of marriage on the division of household labor: a life-course perspective». Journal of Marriage and the Family, 58, 78-90.

PRESSER, H. (1994). «Employment schedules among dual-earner spouses and the division of household labor by gender». American Sociological Review, 59, 348-364.

SÁNCHEZ, L. (1994). "Gender, labor allocations, and the psychology of entitlement within the home». Social Forces, vol. 73, 2, 533-553.

SÁNCHEZ, L. y Thomson, E. (1997). "Becoming mothers and fathers: Parenthood, gender and the division of labor». Gender and Society, vol. 11, 6, 747-772.

SHeLTON, B. A. y John, D. (1996). «The division of household labor». Annual Review of Sociology, 22, 299-322.

SOUTH, S. y SPITZE, G. (1994). «Housework in marital and nonmarital households». American Sociological Review, 59, 327-347.

SteVens, D.; KigeR, G. y RiLEY, P. (2001). «Working hard and hardly working: domestic labor and marital satisfaction among dual-earner couples». Journal of Marriage and Family, 63, 514-526.

SubiRATS, M. (1993). «El trabajo doméstico, nueva frontera para la igualdad». En: GARRIDO, L. y GIL, E. (eds.). Estrategias familiares. Madrid: Alianza, 299-315.

Sullivan, O. (2000). "The division of domestic labour: twenty years of change?». Sociology, vol. 34, 3, 437-456.

- (2004). "Changing gender practices within the household. A theoretical perspective». Gender and Society, vol. 18, 2, 207-222.

Tobío Soler, C. (1998). «Roles de género y la relación familia-empleo». Asparkia: Investigación Feminista, 9, 21-44.

- (2005). Madres que trabajan: Dilemas y estrategias. Madrid: Cátedra.

TORNS, T.; BORRÁS, V. y CARRASQUER, P. (2003-2004). «La conciliación de la vida laboral y familiar: ¿un horizonte posible?». Sociología del Trabajo, 50, 111-137.

TwigGs, J.; MCQUillan, J. y MarX FerREE, M. (1999). «Meaning and measurement: reconceptualising measures of the division of household labor». Journal of Marriage and the Family, vol. 61, 3, 712-724.

VALIENTE, C. (1997). "¿Algo más que "ganadores de pan”?: El papel de los hombres en el ámbito familiar en España (1975-1996)». Revista Española de Investigaciones Sociológicas (REIS), 79, 221-246.

VALLES, M. S. (1997). Técnicas cualitativas de investigación social: Reflexión metodológica y práctica profesional. Madrid: Síntesis.

Zvonkovic, A.; Greaves, K.; SChMiege, C. y Hall, L. (1996). «The marital construction of gender through work and family decisions: a qualitative analysis». Journal of Marriage and the Family, 58, 91-100. 